\title{
Acoustical Properties of Ternary Mixtures of Di-(2ethyl- hexyl)phosphoric Acid, Benzene and $m$-Xylene at Different Temperatures
}

\author{
M. MISHRA ${ }^{1}$, N. SWAIN ${ }^{2}$, B. DALAI ${ }^{3}$, S. K. SINGH ${ }^{4}$ and U. N. DASH ${ }^{1 *}$ \\ ${ }^{1}$ Department of Chemistry, Institute of Technical Education and Research, SOA University, \\ Bhubaneswar-751 030, Odisha, India \\ ${ }^{2}$ Department of Chemistry, College of Basic Science and Humanities, OUAT, \\ Bhubaneswar-751 003, Odisha, India \\ ${ }^{3}$ Department of Physics, Eastern Academy of Science and Technology, \\ Bhubaneswar-754 001, Odisha, India \\ ${ }^{4}$ Department of IMMT, Institute of Minerals and Materials Technology (CSIR), \\ Bhubaneswar-751 013, Odisha, India \\ manaswinimishra15@gmail.com
}

Received 9 April 2014 / Accepted 2 May 2014

\begin{abstract}
Ultrasonic velocity (U) and density $(\rho)$ of the ternary mixtures of di-(2-ethylhexyl) phosphoric acid (DEHPA), benzene and m-xylene were measured at atmospheric pressure and temperatures from $298.15 \mathrm{~K}$ to $313.15 \mathrm{~K}$ at an interval of $5 \mathrm{~K}$. The experimental data have been used to calculate adiabatic compressibility $\left(K_{s}\right)$, intermolecular free length $\left(L_{f}\right)$, acoustic impedance $(Z)$, surface tension $(\sigma)$, molar volume $\left(V_{m}\right)$ and Wada's constant $(W)$. From the data, the deviations in adiabatic compressibility $\left(\Delta K_{s}\right)$, ultrasonic velocity $(\Delta U)$, intermolecular free length $\left(\Delta L_{f}\right)$ and acoustic impedance $(\Delta Z)$ were calculated. The results are discussed in terms of molecular interaction among components of the ternary mixture. The theoretical values of ultrasonic velocity have been calculated using various empirical relations and are compared with the corresponding experimental data by calculating percentage deviation.
\end{abstract}

Keywords: DEHPA, Ternary mixture, Ultrasonic studies, Molecular interaction

\section{Introduction}

Molecular interaction study ${ }^{1-3}$ plays an important role in understanding the structure and properties of liquids. A large number of studies have been made on the interaction in liquid mixture by various methods like dielectric relaxation, Infrared spectroscopy, nuclear magnetic resonance spectroscopy etc. have become powerful tools to provide information regarding the physical and chemical properties of liquid system. The method of studying the molecular interaction from the knowledge of variation of thermodynamic parameters with 
composition and temperature gives an insight into the molecular process. Industry demands reliable data on the physical and chemical properties of a wide variety of liquid mixtures. DEHPA is one of the most widely used and characterized extractant ${ }^{4}$ in the atomic energy industry. Increasing use of DEHPA, benzene and m-xylene in many industrial process have greatly stimulated the need for extensive information on the acoustic and transport properties of these liquids and their mixtures. DEHPA is a polar liquid and the dipolar association of DEHPA molecules decreases when they are mixed with non-polar aromatic hydrocarbons, viz. benzene and m-xylene due to some specific intermolecular interaction between DEHPA molecules and aromatic hydrocarbons.

In the present study, ultrasonic velocities in the ternary mixtures have been estimated using impedance dependence relation ${ }^{5}$, Nomoto's relation ${ }^{6}$, and Rao's specific sound velocity relation ${ }^{7}$. The validity of these formulations for describing the ultrasonic response in this present study has been compared with experimental values by calculating percentage deviation. Such comparisons help to understand the thermodynamics of the mixtures.

\section{Experimental}

All chemicals are of AR grade (E. Merck, India) and the purity of the sample was checked by comparing the experimental data of density and ultrasonic velocity with the values available in the literature ${ }^{8-11}$. The ternary liquid mixture of DEHPA, benzene and m-xylene were prepared by volume. Nine mixtures were prepared in air tight bottles. The mass measurements were performed on a digital top loading balance with a precision of $0.0001 \mathrm{~g}$. Density of pure liquids and their mixtures were determined with a specific gravity bottle of $25 \mathrm{~mL}$ capacity calibrated at four different temperatures from $298.15 \mathrm{~K}$ to $313.15 \mathrm{~K}$. The increment in temperature was kept regular at $5 \mathrm{~K}$, with a view to ensuring measurable effects of temperature change on experimental observations. The maximum error in the density measurement was found to be $\pm 0.01 \mathrm{~kg} \mathrm{~m}^{-3}$. The ultrasonic velocity was determined by using a single crystal variable path ultrasonic interferometer (F-81, Mittal Enterprises, New Delhi) working at $2 \mathrm{MHz}$ with an accuracy of $0.5 \mathrm{~ms}^{-1}$. The working principle used in the measurement of speed of sound through the sample was based on the accurate determination of the wavelengths of ultrasonic waves of known frequency produced by quartz crystal in the measuring cell. The temperature of the sample was controlled by circulating water at a desired temperature through a steel jacket of double walled cell. The temperature of the sample was maintained to a precision of $\pm 0.1 \mathrm{~K}$ in an electronically digital operated water bath for measurement of all the physical properties.

\section{Theoretical}

The experimental values of $U$ and $\rho$ were taken to calculate the various thermo-acoustic parameters, viz. adiabatic compressibility $\left(K_{s}\right)$, intermolecular free length $\left(L_{f}\right)$, acoustic impedance $(Z)$, surface tension $(\sigma)$, molar volume $\left(V_{m}\right)$ and Wada's constant $(W)$, using following relations ${ }^{11-13}$ and are presented in Table 1 .

$$
\begin{gathered}
K_{s}=\frac{1}{\rho U^{2}} \\
L_{f}=k K_{s}^{1 / 2}
\end{gathered}
$$

Where $k\left[=(93.875 \pm 0.375 T) \times 10^{-8}\right]$ is the Jacobson temperature dependent constant.

$$
Z=\rho U
$$




$$
\begin{aligned}
\sigma & =6.3 \times 10^{-4} \rho U^{2} \\
V_{m} & =\sum_{i=1}^{3} x_{i} M_{i} / \rho \\
W & =V_{m} K_{s}^{-1 / 7}
\end{aligned}
$$

The excess function $(\Delta \mathrm{Y})$ such as $\Delta U, \Delta Z, \Delta K_{s}$ and $\Delta L_{f}$ have been computed using the following expression.

$$
\Delta \mathrm{Y}=\mathrm{Y}_{\text {exp }}-\mathrm{Y}_{\text {ideal }}=\mathrm{Y}_{\mathrm{m}}-\left(x_{1} \mathrm{Y}_{1}+x_{2} \mathrm{Y}_{2}+x_{3} \mathrm{Y}_{3}\right)
$$

Where $\mathrm{Y}_{\mathrm{m}}$ represents the parameters $Z, K_{s}, L_{f}, V m$ of ternary mixtures, $x$ is the mole fraction, and subscripts 1, 2 and 3 refer to components of ternary mixture. Using Impedance dependence relation ${ }^{5}$, Nomoto's relation ${ }^{6}$ and Rao's specific sound velocity relation ${ }^{7}$, theoretical values of ultrasonic velocity such as $U_{I D R}, U_{N}$ and $U_{R}$ respectively were estimated (Table 3 ) by the following relations and models as given by :

$$
\begin{aligned}
& U_{I D R}=\sum x_{i} Z_{i} / \sum x_{i} \rho_{i} \\
& U_{N}=\left(\sum x_{i} R_{i} / \sum x_{i} V_{m i}\right)^{3} \\
& U_{R}=\left(\sum x_{i} r_{i} \rho\right)^{3}
\end{aligned}
$$

Where $x_{i}, \rho_{i}, Z_{i}, R_{i}=V_{m i} U_{i}^{1 / 3}, r_{i}=U_{i}^{1 / 3} / \rho_{i}$ and $V_{m i}$ are mole fraction, density, acoustic impedance, molecular sound velocity, Rao's specific sound velocity and molar volume of $i^{\text {th }}$ component of the mixture, respectively. The percentage deviation in ultrasonic velocity between experimental and computed values can be calculated as

$$
\left(\frac{\Delta U}{U}\right) \%=\left(\frac{U_{\exp }-U_{t h}}{U_{\exp }}\right) \times 100
$$

\section{Results and Discussion}

The comparison between experimental and literature values of density $(\rho)$ and ultrasonic velocity (U) of pure liquids are given in the Table 1 .

Table 1. Density $(\rho)$ and ultrasonic velocity $(\mathrm{U})$ of pure liquids at different temperatures

\begin{tabular}{cccccc}
\hline & \multicolumn{3}{c}{$\rho, \mathrm{kgm}^{-3}$} & \multicolumn{2}{c}{$\mathrm{U}, \mathrm{ms}^{-1}$} \\
\hline Pure Liquid & $\mathrm{T}, \mathrm{K}$ & Expt. & Lit. $^{[\mathrm{Ref}]}$ & Expt. & Lit. $^{[\mathrm{Ref}]}$ \\
\hline \multirow{4}{*}{ DEPHA } & 298.15 & 975.0 & - & 1344 & - \\
& 303.15 & 916.3 & $1009.6^{[21]}$ & 1324 & $1321.6^{[13]}$ \\
& 308.15 & 959.2 & $1008.2^{[21]}$ & 1304 & $1302.2^{[13]}$ \\
& 313.15 & 955.8 & $1003.1^{[21]}$ & 1284 & $1283.4^{[13]}$ \\
Benzene & 298.15 & 873.6 & $873.68^{[8]}$ & 1302 & $1299.3^{[20]}$ \\
& 303.15 & 868.2 & $868.26^{[20]}$ & 1276 & $1276.0^{[20]}$ \\
& 308.15 & 862.8 & $862.89^{[20]}$ & 1254 & $1252.8^{[20]}$ \\
& 313.15 & 854.4 & $857.49^{[20]}$ & 1230 & $1229.8^{[20]}$ \\
m-Xylene & 298.15 & 859.8 & $859.87^{[9]}$ & 1321.5 & $1321.0^{[21]}$ \\
& 303.15 & 855.4 & - & 1303.7 & - \\
& 308.15 & 851.5 & $851.24^{[9]}$ & 1283.5 & $1279^{[9]}$ \\
& 313.15 & 847.2 & - & 1234.3 & - \\
\hline
\end{tabular}


Table 2. Experimentally determined values of density $(\rho)$, ultrasonic velocity $(U)$ and calculated values of isentropic compressibility $\left(K_{s}\right)$, intermolecular free length $\left(L_{f}\right)$, acoustic impedance $(Z)$, surface tension $(\sigma)$, molar volume $\left(V_{m}\right)$ and Wada's constant $(W)$ at different temperatures

\begin{tabular}{|c|c|c|c|c|c|c|c|c|c|c|}
\hline $\bar{x}$ & $\tilde{x}$ & $\tilde{x}$ & $\Omega^{3} \Xi$ & $D T^{\prime}$ & 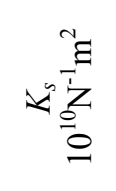 & $\sqrt{\Xi+}$ & 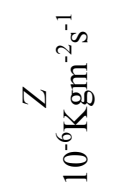 & 话 & 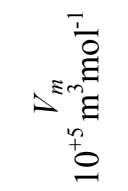 & 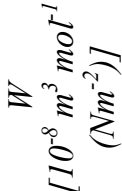 \\
\hline \multicolumn{11}{|c|}{$298.15 \mathrm{~K}$} \\
\hline 0.034 & תנת & +00 & 4.2 & 1236 & 7.4847 & 5.6282 & 0805 & t.1Jנ & 1.2038 & \\
\hline 0.072 & & & 1.2 & 1243 & 7.3465 & & 9950 & & .0922 & \\
\hline 0.124 & 0.506 & 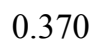 & 894.1 & 1249 & & & & 87.872 & & \\
\hline & 0.471 & 0.343 & 900.1 & 1253 & 7.0763 & & & & & 3001 \\
\hline & 0434 & 031 & 911.2 & 1256 & & & & & & 3304 \\
\hline & 038 & 0.2 & 9 & 1263 & & & & & & 3706 \\
\hline & 0 & & .2 & 1269 & & & & & & \\
\hline & & & 3 & 1274 & & & & & & \\
\hline & & & & 1299 & & 5.1226 & & & & \\
\hline \multicolumn{11}{|c|}{$303.15 \mathrm{~K}$} \\
\hline & & & & & 535 & & & & & \\
\hline & & & & & & & & & & \\
\hline & & & & & & & & & & \\
\hline & & & & & & & & & & \\
\hline & & & & & & & & & & \\
\hline & & & & & & & & & & \\
\hline & & & & & & & & & & \\
\hline & & & & & & & & & & \\
\hline & 163 & & & 1282 & & 392 & 41 & & & \\
\hline \multicolumn{11}{|c|}{$308.15 \mathrm{~K}$} \\
\hline 0.034 & & & & & 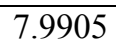 & & & & & \\
\hline & & & & & & & & & & \\
\hline & 0.506 & & & 1215 & & & & & & \\
\hline & 471 & & & 1219 & & & & & & \\
\hline & & & & & & & & & & \\
\hline & & & & & & & & & & \\
\hline & & & & & & & & & & \\
\hline & & & & & & & & & & \\
\hline & & & & & & 5.3973 & & & & \\
\hline \multicolumn{11}{|c|}{$313.15 \mathrm{~K}$} \\
\hline & & & & & 614 & 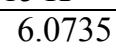 & & & & \\
\hline & 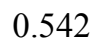 & & 6 & 4 & & & & & & 138 \\
\hline & $-5 r$ & & 5.2 & 1197 & & & & & & 580 \\
\hline & 471 & 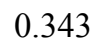 & .5 & 1202 & & & & & 22 & \\
\hline & 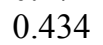 & & 1.5 & 1205 & & & & & & \\
\hline & & & & 1213 & & & & & & \\
\hline & & & & 1221 & & & & & & \\
\hline & & & 933.2 & 1229 & & & & & & 0.4752 \\
\hline 729 & 163 & 108 & 947.9 & 1235 & 6.7195 & 5.4774 & 1.1877 & 93.7573 & 27.3499 & 0.5589 \\
\hline
\end{tabular}


The measurement of ultrasonic velocity in ternary mixture provides some reliable information in the study of molecular interaction. The isentropic compressibility $\left(K_{s}\right)$, intermolecular free length $\left(L_{f}\right)$, acoustic impedance $(Z)$, surface tension $(\sigma)$, molar volume $\left(V_{m}\right)$ and Wada's constant $(W)$ for ternary mixture, DEHPA $\left(\mathrm{x}_{1}\right)+$ benzene $\left(\mathrm{x}_{2}\right)+\mathrm{m}$-xylene $\left(\mathrm{x}_{3}\right)$ at different temperatures are listed in Table 2. The deviation values, viz. $\Delta K_{s}, \Delta L_{f}, \Delta Z$ and $\Delta U$ of the ternary mixture have been calculated and displayed in Figures 2-5.

From Table 2, it is observed that the values of ultrasonic velocity $(\mathrm{U})$ and density $(\rho)$ increase nonlinearly with increase in DEHPA mole fraction $\left(\mathrm{x}_{1}\right)$. Table 2 shows that the density and ultrasonic velocity are in increasing trend with increase in mole fraction of DEHPA. According to Arul et al. ${ }^{14}$, the increase in ultrasonic velocity in any mixture indicates the association among molecules of a mixture. It is observed from Table 2 that the values of $K_{s}$ and $L_{f}$ decrease with mole fraction of DEHPA and increases with increase in temperature. The rapid decrease in $K_{s}$ with increase in concentration of DEHPA indicates the formation of large number of tightly bound systems. According to Pandey et $a .^{15}$, as velocity and density increase with concentration, isentropic compressibility $\left(K_{s}\right)$ must decrease with increase in concentration, as they vary inversely with each other. The free length is the distance between the surfaces of the neighboring molecules. It indicates significant interactions between the solute and solvent molecules, due to which the structural arrangement in the neighborhood of constituent solute particles is considerably affected. Values of acoustic impedance $(Z)$, surface tension $(\sigma)$, molar volume $\left(V_{m}\right)$ and Wada's constant $(W)$ show a nonlinear increasing trend with increasing mole fraction of DEHPA. According to the model proposed by Eyring and Kincaid ${ }^{16}$, the trend of variation of ultrasonic velocity and isentropic compressibility (intermolecular free length) is opposite which corroborates our findings. Furthermore, the nonlinear increase in the value of $Z, \sigma, V_{m}$ and $W$ with composition of DEHPA lends support to the interaction between the component molecules.

A continuous decrease in $K_{s}$ and $L_{f}$, and increase of acoustic impedance with concentration at the experimental temperatures are clear evidence for the existence of strong interactions like dipole-dipole, dipole-induced dipole, formation of charge transfer complex etc.

It is observed that the increasing trend of Wada's constant $(W)$ and surface tension $(\sigma)$ with mole fraction of DEHPA indicates strong solute - solvent interactions ${ }^{13}$. The dimeric structure of DEHPA molecule (Figure 1) is characterized by intermolecular hydrogen bonding between $\mathrm{OH}$ group of one molecule with $\mathrm{P}=\mathrm{O}$ of the other molecule.

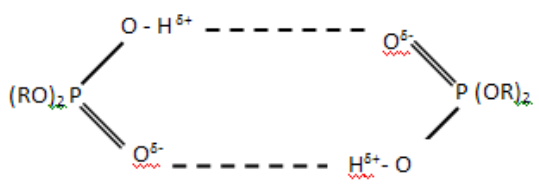

where $\mathrm{R}$ is ethylhexyl.

Figure. 1. Dimeric form of DEHPA

The simultaneous participation of both groups affects the extraction ability. As benzene and m-xylene are ring type structure and they are non polar, the attractive interactions are less, because DEHPA is polar and other two (benzene \& m-xylene) are non-polar and each component maintains its identity. Thus molar volume $V_{m}$ between the components is in an increasing trend with increase in the mole fraction of DEHPA. This reduces the net attractive forces.

The deviation in the physical property from its ideal behaviour is a measure of the degree of interaction between component molecules of the ternary liquid mixture. The deviation parameters play a major role in understanding the nature of molecular interactions 
in liquid mixtures. The various types of solute-solvent interactions ${ }^{17}$ that are operating between the unlike molecules are dispersive forces, which make a positive contribution to deviation parameters and hence H-bonding, dipole-dipole interactions, and dipole-induced dipole interactions are expected to make a negative contribution. The interstitial accommodation which regulates the voids in the liquid structure might be a regulating factor in the magnitudes of deviation parameters.

The deviations in intermolecular free length, isentropic compressibility, $\Delta K_{s}$ (Figure 2) and $\Delta L_{f}$ (Figure 3 ) are positive throughout the whole range of composition of DEHPA in the ternary mixture. On the other hand, the deviations in acoustic impedance, $\Delta Z$ (Figure 4 ) and ultrasonic velocity $\Delta U$ (Figure 5) show the inverse trend to that of $\Delta K_{s}$ and $\Delta L_{f}$. According to Fort and Moore ${ }^{18}$, positive values of deviation functions correspond to the existence of dispersive force. Positive value of $\Delta K_{s}$ is associated with a structure breaking tendency due to hetero-molecular interaction between the component molecules of the mixtures. The shape and size of the molecules in the mixture are loosely packed which is due to the positive excess isentropic compressibility. In this ternary system, the positive deviation in $\Delta K_{s}$ and $\Delta L_{f}$ with concentration and temperatures have been attributed to dispersive forces that show weak interaction between the unlike molecules. This may be due to possible $\pi \cdots \cdots \pi$ interaction between $\pi$. electrons of benzene and m-xylene with functional groups of DEHPA.

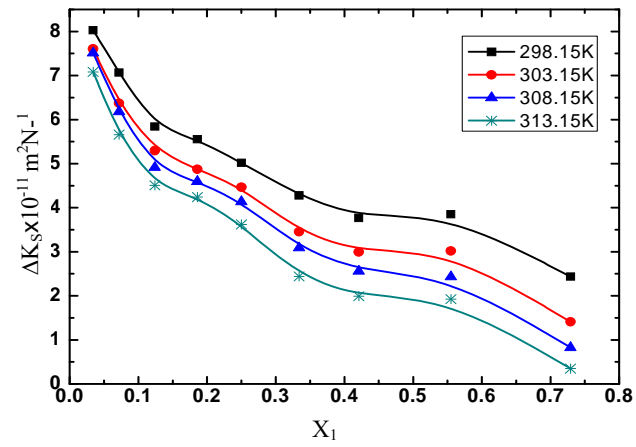

Figure 2. Deviation in isentropic compressibility $\left(\Delta K_{S}\right) v s$. molefraction $\left(X_{1}\right)$ of DEHPA

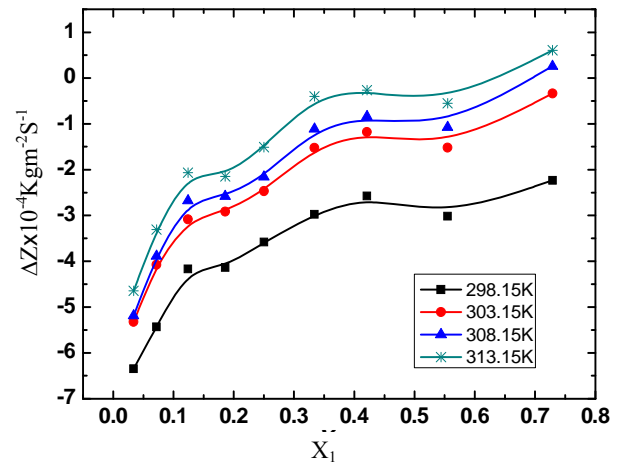

Figure 4. Deviation in acoustic impedance $(\Delta Z)$ vs. molefraction $\left(X_{1}\right)$ of DEHPA

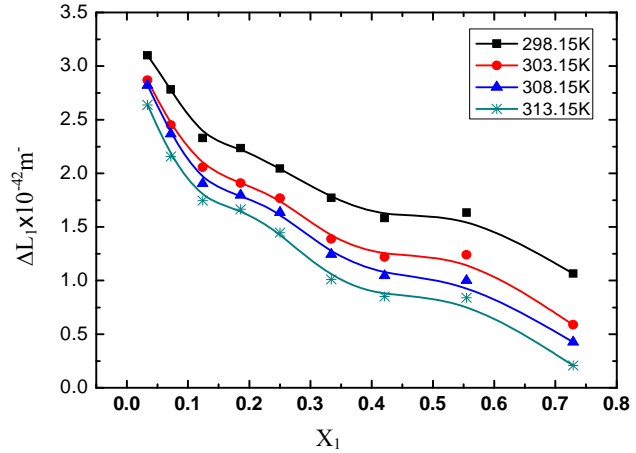

Figure 3. Deviation in intermolecular free length $\left(\Delta L_{f}\right)$ vs. molefraction $\left(X_{1}\right)$ of DEHPA

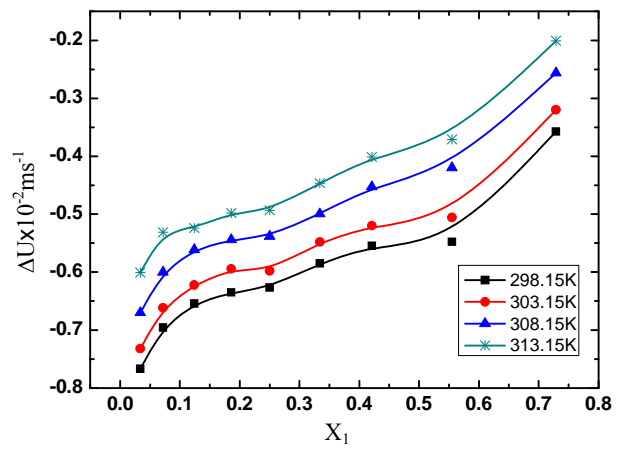

Figure 5. Deviation in ultrasonic velocity $(\Delta U) v s$. mole fraction $\left(X_{1}\right)$ of DEHPA 
The values of $\Delta U$ and $\Delta Z$ are negative over entire composition range at four different temperatures. Negative deviation values suggest the presence of weak interaction between the component molecules ${ }^{11,13}$. The deviation in ultrasonic velocity may be explained in terms of relative molecular interactions between like and unlike molecules. In the present case, the mutual loss of dipolar association among DEHPA molecules would contribute to negative $\Delta U$ indicating dispersive interaction among the components. The variation of $\Delta \mathrm{Z}$ follows similar trend as deviation in ultrasonic velocity for the system at different temperatures which agrees well with the results of $\Delta K_{s}$ and $\Delta L_{f}$. Positive values of $\Delta K_{s}, \Delta L_{f}$ and negative values of $\Delta Z, \Delta U$ in the ternary mixture indicate the presence of weak molecular interactions among the components of molecules in the mixture.

The observed deviations of the theoretical ultrasonic velocity from the experimental values are attributed to the presence of intermolecular interactions ${ }^{19}$ between the component molecules of the mixture. The observed deviations (Table 3) following all theories illustrate molecular interaction between unlike molecules in the liquid mixtures. The suitability of these theories based on percentage deviation value is $U_{I D R}>U_{N}>U_{R}$.

Table 3. The Comparison between experimental and theoretical values of ultrasonic velocity with mole fraction $\left(x_{1}\right)$ of DEHPA made at four different temperatures, but is given at a single temperature $(303.15 \mathrm{~K})$

\begin{tabular}{cccccccc}
\hline & \multicolumn{4}{c}{ Ultrasonic Velocity, $\mathrm{c}$} & \multicolumn{3}{c}{ Percentage deviation, $(\Delta U / U) \%$} \\
\cline { 2 - 8 } $\begin{array}{c}\text { Mole } \\
\text { raction, } \\
\mathrm{x}_{1}\end{array}$ & $\begin{array}{c}U_{\text {Exp. }} \\
\mathrm{m} / \mathrm{s}\end{array}$ & $\begin{array}{c}U_{I D R} \\
\mathrm{~m} / \mathrm{s}\end{array}$ & $\begin{array}{c}U_{N} \\
\mathrm{~m} / \mathrm{s}\end{array}$ & $\begin{array}{c}U_{R} \\
\mathrm{~m} / \mathrm{s}\end{array}$ & $\begin{array}{c}U_{I D R} \\
\mathrm{~m} / \mathrm{s}\end{array}$ & $\begin{array}{c}U_{N} \\
\mathrm{~m} / \mathrm{s}\end{array}$ & $\begin{array}{c}U_{R} \\
\mathrm{~m} / \mathrm{s}\end{array}$ \\
\hline 0.034 & 1217 & 1273.9 & 1293.2 & 1311.5 & -4.7 & -6.3 & -7.8 \\
0.072 & 1224 & 1272.7 & 1296.4 & 1338.6 & -4.0 & -5.9 & -9.4 \\
0.124 & 1230 & 1271.3 & 1300.3 & 1368.5 & -3.4 & -5.7 & -11.1 \\
0.186 & 1235 & 1269.5 & 1304.1 & 1371.5 & -2.8 & -5.6 & -11.3 \\
0.250 & 1237 & 1267.7 & 1307.3 & 1394.9 & -2.5 & -5.7 & -12.8 \\
0.334 & 1245 & 1265.4 & 1310.7 & 1419.6 & -1.6 & -5.3 & -14.0 \\
0.421 & 1251 & 1263.0 & 1313.6 & 1428.2 & -1.0 & -5.0 & -14.2 \\
0.555 & 1257 & 1259.3 & 1317.0 & 1417.1 & -0.2 & -4.8 & -12.7 \\
0.729 & 1282 & 1254.9 & 1320.3 & 1401.8 & 2.1 & -3.0 & -9.3 \\
\hline
\end{tabular}

\section{Conclusion}

The values of density and ultrasonic velocity of the ternary mixture containing DEHPA, benzene and m-xylene are reported at atmospheric pressure and temperatures from $298.15 \mathrm{~K}$ to $303.15 \mathrm{~K}$. From these data, several acoustical parameters were calculated. The values of $\Delta K_{s}$ and $\Delta L_{f}$ are found to be positive whereas $\Delta Z$ and $\Delta U$ are negative for the ternary mixture. All these parameters indicate the presence of specific interactions between the component molecules. Again, the computed ultrasonic velocities from different theories have been correlated with the experimentally measured values. The results indicate that IDR relation predicts the experimental data well, showing smallest deviations in comparison to other two theories.

\section{Acknowledgement}

The authors are grateful to the Chairman, Institute of Technical Education and Research (ITER), SOA University, Bhubaneswar for providing the necessary facilities to carry out the research work. 


\section{References}

1. Swain N, Singh S K, Panda D and Chakravortty V, J Mole Liq., 2001, 94, 233.

2. Swain N, Panda D, Singh S K and Chakravortty V J, J Chem Engg Data, 1999, 44, 32.

3. Das J K, Dash S K, Swain N and Swain B B, J Mol Liq., 1999, 81, 163.

4. Lee M S, Ahn J G and Lee E C, Hydrometallurgy, 2002, 63, 269.

5. Kumar D S and Rao D K, Ind J Pure Appl Phys., 2007, 45, 210-220.

6. Nomoto O, J Phys Soc Jpn., 1958, 13, 1528-1532.

7. Rao M R, J Chem Phy., 1941, 9, 682-686.

8. Mariano A, Mussari L, Camacho A, Canzonieri and Postigo M, Phys Chem Liq., 2013, 51, 731-741.

9. Bhatia S C, Rani R and Bhatia R, J Mol Liq., 2011, 159, 132-141.

10. Lide D R and Haynes W M, CRC Handbook of Chemistry and Physics, $91^{\text {st }}$ Edn., CRC Press, Boca Raton 2010-2011.

11. Dalai B, Dash S K and Singh S K, Ind J Pure Appl Phys., 2014, 52, 24-29.

12. Dash S K, Pradhan S K, Dalai B, Moharana L and Swain B B, Phys Chem Liq., 2012, 50, 735-749.

13. Mishra S and Paikaray R, Res J Physical Sci., 2013, 1, 15-21.

14. Arul G and Palaniappan, Indian J Pure Appl Phys., 2001, 39, 561-564.

15. Pandey J D, Shukla S A K and Singh A K, Pramana, 1994, 43, 353-360.

16. Kincaid J F and Eyring H, J Chem Phys., 1938, 6, 620-629.

17. Sastry S S, Babu S, Vishwam T and Tiong H S, J Chem Thermodyn, 2014, 68, 183-192.

18. Fort R J and Moore W R, Trans Faraday Soc., 1966, 62, 1112.

19. Ali A, Nain A K, Sharma V K and Ahmad S, Phys Chem Liq., 2004, 42 , 375-383.

20. Malek N I, Ijardar S P, Master Z R and Oswal S B, Thermochemica Acta, 2012, 539, 71-83.

21. Swain N, Chakravorthi V, Singh S K and Panda D, Indian J Chem., 1999, 38A, 1116-1122. 